\title{
Böick, Marcus: Die Treuhand. Idee - Praxis - Erfahrung. 1990-1994, 767 S., Wallstein, Göttingen 2018.
}

\author{
Alexander Leipold \\ Online publiziert: 2. Oktober 2020 \\ (C) Der/die Autor(en) 2020
}

Die Gründung der Treuhandanstalt jährt sich 2020 zum 30. Mal. Anhaltend hohe Arbeitslosigkeit, Produktivitätsdefizite und eine beispiellose demografische Entwicklung im Zuge vereinigungsbedingter (Arbeits-)Migration bieten Anlass genug, sich mit der Geschichte dieser Organisation zu befassen. Das Bundesarchiv hat nach Aktenfreigabe eine Systematisierung der Bestände angekündigt. Und das Institut für Zeitgeschichte wird in mehreren Teilprojekten Detailstudien vorlegen. Marcus Böick hat in seiner 2018 publizierten Dissertation eine Vorlage geschaffen, die hierfür maßgeblich sein wird. Der mehr als 700 Seiten starke Band kann als ,Totalgeschichte der Treuhand verstanden werden. Die von Böick vorgelegte Synthese verbindet nebeneinanderliegende Debattenstränge aus Geschichts- und Sozialwissenschaften, reichert diese mit eigenen Zeitzeugeninterviews an und widmet sich der Frage, wie der wirtschaftliche Systemwechsel ,konzeptionell erdacht, alltäglich gestaltet und individuell reflektiert wurde " [Hervorh. im Orig.] (S. 17).

Böick zieht unveröffentlichte Quellen aus dem Bundesarchiv, dem Parlamentsarchiv des Deutschen Bundestages sowie einiger Privatarchive heran. Die Rekonstruktion der Organisationsgeschichte stützt sich maßgeblich auf das noch von der Treuhand veröffentlichte Medienarchiv. Wirtschaftswissenschaftliche Debattenbeiträge und sozialwissenschaftliche Transformationsliteratur der 1990er Jahre unterwirft der Autor einer konsequenten Historisierung. Dadurch werden Zeit- und Kontextgebundenheit der damaligen Annahmen offenbar. Besonderen Wert hat der Rückgriff auf drei Interviewreihen, von denen zwei noch während der ,heißen' Phase der Treuhand (1992-1994) durchgeführt wurden. Eine dritte, vom Autor zur Ergänzung durchgeführte Interviewreihe muss aufgrund ihres Entstehungszeitpunktes 20 Jahre später auch als historisches Komplement verstanden werden. Denn die Reflexionen aus erster Hand geben der unteren Hierarchieebene Gelegenheit zum Rückblick. Die Debattenrekonstruktion fördert eine Unmenge an Akteuren zutage, deren wechselseitige Verschränkung teilweise ins Unüberschaubare gerät. Chronologien, Tabellen oder Schaubilder hätten die Komplexität der Ereignisse für den Leser greifbar machen können.

\footnotetext{
A. Leipold $(\bowtie)$

Leuphana Universität Lüneburg, Lüneburg, Deutschland

E-Mail: alexander.leipold@leuphana.de
} 
Der Autor verfolgt seinen Beweisgang in drei Abschnitten, beginnend mit den konzeptionellen Grundlagen der ökonomischen Transformation im Zuge des Vereinigungsprozesses. Der als „Ideen- und Konzeptgeschichte“ überschriebene Teil von rund 140 Seiten behandelt rivalisierende Konzepte der (westdeutschen) Wirtschaftswissenschaft und legt den Eigensinn der Bonner Ministerialbürokratie offen. Diese hatte sich in ihrer Not eines Essays von Ludwig Erhard aus dem Jahre 1953 als Blaupause und Legitimation für das von ihr vorgestellte Transformationskonzept bedient (S. 153). Dieses Detail verdient Erwähnung, nicht nur, weil die Beamten um Thilo Sarrazin und Hans Tietmeyer damit Erhards Skepsis gegenüber wissenschaftlicher Planbarkeit der Wirtschaft wiederholten, sondern weil Böick im Schlussteil eine Verortung der Treuhand in der neoliberalen Programmatik verneint (S. 726). Was aber, wenn nicht die auch in der Ministerialbürokratie geteilten Ansichten eines überzeugten Neoliberalen könnten hierfür maßgeblich sein?

Dem schließen sich ein etwa 300 Seiten umfassender Teil zur „Organisationsund Praxisgeschichte“ sowie die als „Sozial- und Erfahrungsgeschichte“ behandelte Auswertung der Zeitzeugeninterviews (etwa 250 Seiten) an. Beide Teile sollten aufeinander bezogen gelesen werden. Maßgeblich vorangetrieben wurde die Organisationspraxis Böick zufolge von vier Typen. Allen voran waren dies westdeutsche Industriemanager, deren Anwerbung Treuhand-Chef Detlev Rohwedder ab August 1990 forcierte. Die Praktiker legten einen nahezu blinden Glauben an die unternehmerische Schaffenskraft an den Tag, dem auch die erste und letzte frei gewählte Volkskammer und die Regierung de Maizière folgten. Sie wurden von einer bunten Schar von Verwaltungsexperten („Bürokraten unter Managern“) unterstützt. Hierbei handelte es sich um Verwaltungsjuristen, altgediente Ministerialnomenklatur und vormalige Leiter öffentlich-rechtlicher Unternehmen, die ihre bürokratische Sozialisation im Westen erfuhren und nun an der Schnittstelle von Politik und Wirtschaft um ihre Position rangen. Böick spricht von ,exotischen Grenzlanderfahrungen“(S. 619).

Avers verhielten sich die einstigen Planwirtschaftskader, die nicht nur ein anderes Verständnis von politischer Ökonomie mitbrachten, sondern nun auch den ,eigenen Leuten ' die zahlreichen und dann unter Rohwedders Nachfolgerin, Birgit Breuel, mit Hochdruck forcierten Werkschließungen vermitteln mussten. Die zitierten Interviews legen nahe, dass aus diesem Spagat bis heute nachwirkende Wunden resultieren. Mehr als Randgruppe vorgestellt werden schließlich die nominell überrepräsentierten, aber zumeist nur in Hilfsfunktionen beschäftigten Frauen der Treuhand. Deren marginale Position trug zu einer Reproduktion von Geschlechterungleichheiten bei, die durch ost- und westdeutsche Identitätszuschreibungen noch verstärkt wurden. Damit sei die Treuhand, so Böick, getreues Abbild westdeutscher Behörden und Unternehmen gewesen (S. 693). Ein Umstand, den die befragten Führungskräfte teilweise reflektierten, der jedoch im Hinblick auf eine genuin ostdeutsche Perspektive auf der ,Arbeitsebene' weiterhin der Aufarbeitung harrt.

Das Buch nimmt keine politische Einordnung der Treuhand vor. Es ist auch nicht seine Zielsetzung. Der Disput um die wirtschaftlichen Folgen der Wiedervereinigung muss im gesellschaftlichen Diskurs geklärt werden. Böick gibt dazu mit seiner Geschichte der Treuhand ein instruktives Kompendium an die Hand, das vor allem die Eigenlogik der Behörde und den situativen Rückgriff ihrer Akteure auf wirtschaftspolitische Ideen aufzeigt. 
Funding Open Access funding enabled and organized by Projekt DEAL.

Open Access Dieser Artikel wird unter der Creative Commons Namensnennung 4.0 International Lizenz veröffentlicht, welche die Nutzung, Vervielfältigung, Bearbeitung, Verbreitung und Wiedergabe in jeglichem Medium und Format erlaubt, sofern Sie den/die ursprünglichen Autor(en) und die Quelle ordnungsgemäß nennen, einen Link zur Creative Commons Lizenz beifügen und angeben, ob Änderungen vorgenommen wurden.

Die in diesem Artikel enthaltenen Bilder und sonstiges Drittmaterial unterliegen ebenfalls der genannten Creative Commons Lizenz, sofern sich aus der Abbildungslegende nichts anderes ergibt. Sofern das betreffende Material nicht unter der genannten Creative Commons Lizenz steht und die betreffende Handlung nicht nach gesetzlichen Vorschriften erlaubt ist, ist für die oben aufgeführten Weiterverwendungen des Materials die Einwilligung des jeweiligen Rechteinhabers einzuholen.

Weitere Details zur Lizenz entnehmen Sie bitte der Lizenzinformation auf http://creativecommons.org/ licenses/by/4.0/deed.de. 